\title{
Measuring financial risk and portfolio optimization with a non-Gaussian multivariate model
}

by Young Shin Kim, Rosella Giacometti,

Svetlozar T. Rachev, Frank J. Fabozzi, Domenico Mignacca

No. 44 | AUGUST 2012

\section{WORKING PAPER SERIES IN ECONOMICS}

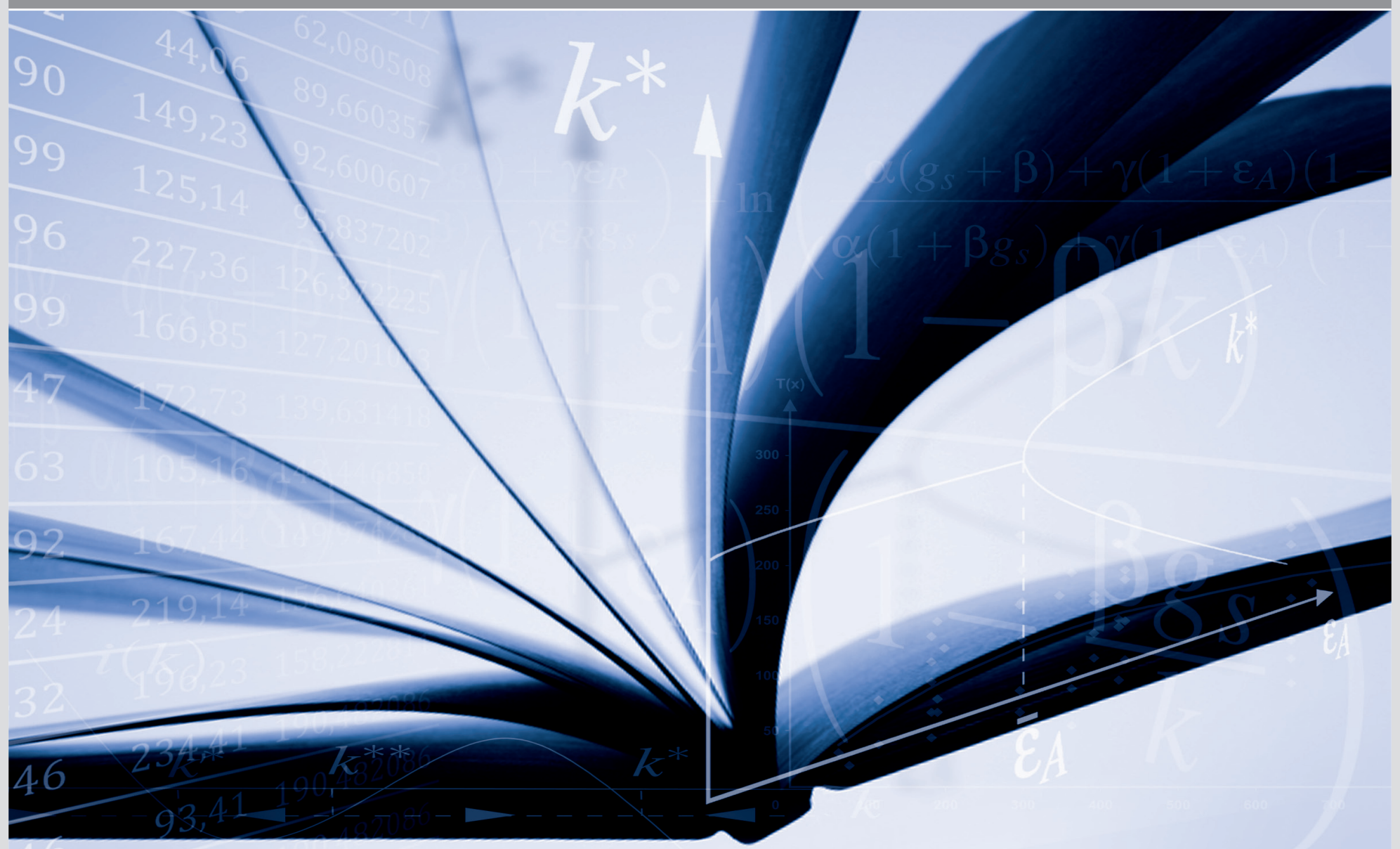




\section{Impressum}

Karlsruher Institut für Technologie (KIT)

Fakultät für Wirtschaftswissenschaften

Institut für Wirtschaftspolitik und Wirtschaftsforschung (IWW)

Institut für Wirtschaftstheorie und Statistik (ETS)

Schlossbezirk 12

76131 Karlsruhe

KIT - Universität des Landes Baden-Württemberg und nationales Forschungszentrum in der Helmholtz-Gemeinschaft

Working Paper Series in Economics

No. 44, August 2012

ISSN 2190-9806

econpapers.wiwi.kit.edu 


\section{Measuring Financial Risk and Portfolio Optimization with a Non-Gaussian Multivariate Model}

August 14, 2012

\section{Young Shin Kim}

Department of Statistics, Econometrics and Mathematical Finance,

School of Economics and Business Engineering, Karlsruhe Institute of Technology, Germany

E-mail: aaron.kim@kit.edu

\section{Rosella Giacometti}

Department of Mathematics, Statistics, Computer Science and Applications

University of Bergamo, Italy

E-mail: rosella.giacometti@unibg.it

\section{Svetlozar T. Rachev}

Frey Family Foundation Professor Department of Applied Mathematics \& Statistics,

Stony Brook University

and

Chief-Scientist, FinAnalytica

E-mail: rachev@ams.sunysb.edu

\section{Frank J. Fabozzi}

EDHEC Business School

Lille, France

E-mail: frank.fabozzi@edhec.edu

\section{Domenico Mignacca}

Eurizon Capital

E-mail: domenico.mignacca@eurizoncapital.com 


\begin{abstract}
In this paper, we propose a multivariate market model with returns assumed to follow a multivariate normal tempered stable distribution. This distribution, defined by a mixture of the multivariate normal distribution and the tempered stable subordinator, is consistent with two stylized facts that have been observed for asset distributions: fat-tails and an asymmetric dependence structure. Assuming infinitely divisible distributions, we derive closed-form solutions for two important measures used by portfolio managers in portfolio construction: the marginal VaR and the marginal AVaR. We illustrate the proposed model using stocks comprising the Dow Jones Industrial Average, first statistically validating the model based on goodnessof-fit tests and then demonstrating how the marginal VaR and marginal AVaR can be used for portfolio optimization using the model. Based on the empirical evidence presented in this paper, our framework offers more realistic portfolio risk measures and a more tractable method for portfolio optimization.
\end{abstract}

JEL Classifications: C58, C61, G11, G32

Keywords: portfolio risk, portfolio optimization, portfolio budgeting, marginal contribution, fat-tailed distribution, multivariate normal tempered stable distribution

\title{
1 Introduction
}

A major contribution to the theory of portfolio theory is the mean-variance model presented by Markowitz (1952). Although some of the assumptions underlying the model have been challenged since its introduction, the importance of the model cannot be overstated. The optimization model has been used for the asset allocation decision, portfolio construction within an asset class, and in asset-liability management. ${ }^{1}$ Alternative models have been proposed based on the relaxation of one or more of the model's assumption. Although a review of all of these extensions is beyond the scope of this paper, we mention two assumptions that are relevant for this paper.

The first is the assumption that asset returns follow a normal (Gaussian) distribution and therefore the use of the variance as a measure of risk ignoring higherorder moments. The assumption of the normality of return distributions has dominated financial theories despite the preponderance of empirical evidence that dates

\footnotetext{
${ }^{1}$ See Fabozzi et al (2002) for a review.
} 
back to Mandelbrot (1963a,1963b) and Fama (1963) who find no support for this assumption, as well as theoretical arguments that suggest return distributions will not be normally distributed. ${ }^{2}$ What studies have found is that asset returns exhibit fat tails and asymmetry. Consequently, non-Gaussian distribution models that can accommodate the observed stylized facts about return distributions have been proposed for use in optimization models for portfolio construction, particularly in the case of equities.

The subordinated Gaussian distribution is a popular model for constructing a multivariate market model that accommodates fat tails and asymmetry. This distribution is defined by taking the multivariate normal distribution and changing the variance to a strictly positive random vector. For example, in Rachev and Mittnik (2000), portfolio analysis is formulated assuming an $\alpha$-stable subordinated Gaussian distribution. Subsequently, the inverse Gaussian subordinated Gaussian distribution (normal inverse Gaussian distribution) and the inverse gamma subordinated Gaussian distribution (skewed- $t$ distribution) have been applied to portfolio analysis by Øigård et al (2005), Aas et al (2006), Eberlein and Madan (2010), Stoyanov et al (2009), and Adcock (2010).

The second assumption, which is related to the first assumption, is the use of portfolio variance as a measure of risk. Alternative risk measures have been proposed. One of the most popular is the value-at-risk (VaR) measure, its popularity being the result of its endorsement by bank regulators despite the wellknown limitations of this measure. A better risk measure that overcomes many of the limitations of VaR and, unlike VaR, is a coherent risk measure is average VaR (AVaR). ${ }^{3}$ The closed-form solution for AVaR for the $\alpha$-stable distribution, the skewed- $t$ distribution, and the infinitely divisible distributions containing tempered stable distributions have been derived by Stoyanov et al (2006), Dokov et al (2008), and Kim et al (2010), respectively. Portfolio optimization using AVaR is also studied in Mansini et al (2007).

An important measure derived from portfolio optimization that managers utilize in making portfolio rebalancing decisions or re-optimization decisions is the marginal risk contribution of a portfolio holding. This risk measure with respect to a given portfolio holding can be thought of as the rate of change in risk (whether it is variance, $\mathrm{VaR}$, or $\mathrm{AVaR}$ ) with respect to a small percentage change in the size of a portfolio holding and is defined by the first derivative of the risk measure with respect to the holding's marginal weight. Because of the importance of this measure in portfolio decisions, a closed-form solution for this measure is needed. The general form of marginal risk contributions with respect to the VaR and AVaR

\footnotetext{
${ }^{2}$ For a review of the empirical evidence, see Rachev et al (2005).

${ }^{3} \mathrm{AVaR}$ is also called conditional value-at-risk (CVaR). AVaR satisfies all axioms of a coherent risk measure and is consistent with preference relations of risk-averse investors. See Pflug (2000), Rockafellar and Uryasev(2000, 2002), and Rachev et al (2007)).
} 
are provided in Gourieroux et al (2000).

In this paper, we propose a multivariate market model with returns assumed to follow a multivariate normal tempered stable (MNTS) distribution. The MNTS distribution, defined by a mixture of the multivariate normal distribution and the tempered stable subordinator, ${ }^{4}$ captures the fat-tail property of asset returns and has an asymmetric dependence structure. We derive closed-form solutions for the marginal VaR and marginal AVaR for infinitely divisible distributions and apply them to the MNTS market model (hereafter simply the MNTS model). We illustrate the procedure using actual stocks, first statistically validating the MNTS model using goodness-of-fit tests and then demonstrating how the marginal VaR and marginal AVaR can be used for portfolio optimization under the MNTS model.

We have organized the paper as follows. The MNTS distribution is presented in Section 2. Closed-form solutions for the marginal VaR and AVaR for infinitely divisible distributions are presented in Section 3, where we also discuss portfolio optimization using the marginal VaR and the marginal AVaR. In Section 4, the market model assuming the MNTS distribution is discussed and the closed-form solutions for the marginal $\mathrm{VaR}$ and $\mathrm{AVaR}$ are applied to the portfolio return for the MNTS model. The empirical illustration for the MNTS model is provided in Section 5 which also reports (1) the empirical results of parameter estimations and the goodness-of-fit tests for the MNTS model and (2) the marginal VaR and AVaR values together with optimal portfolios using those two marginal risks. In Section 6 , we summarize our principal findings. In the paper's appendix, we present the proof of the closed-form solution for the marginal VaR for infinitely divisible distributions.

\section{Multivariate Normal Tempered Stable Distribu- tion}

Let $\alpha \in(0,2)$ and $\theta>0$. The purely non-Gaussian infinitely divisible random variable $T$ whose characteristic function is given by

$$
\phi_{T}(u)=\exp \left(-\frac{2 \theta^{1-\frac{\alpha}{2}}}{\alpha}\left((\theta-i u)^{\frac{\alpha}{2}}-\theta^{\frac{\alpha}{2}}\right)\right)
$$

is referred to as the classical tempered stable (CTS) subordinator with parameters $(\alpha, \theta)$. Let

$$
X=\mu+\beta(T-1)+\gamma \sqrt{T} \varepsilon,
$$

\footnotetext{
${ }^{4}$ The tempered stable subordinated Gaussian process was also studied by Barndorff-Nielsen and Shephard (2001).
} 
where $\mu, \beta \in \mathbb{R}, \gamma>0, \varepsilon \sim N(0,1)$, and $T$ is the CTS subordinator, independent of $\varepsilon$, with parameters $(\alpha, \theta)$. Then, the random variable $X$ is referred to as normal tempered stable (NTS) random variable with parameters $(\alpha, \theta, \beta, \gamma, \mu)$ that we denote by $X \sim \operatorname{NTS}(\alpha, \theta, \beta, \gamma, \mu)$. By composing characteristic functions of $\varepsilon$ and $T$, we obtain the characteristic function of $X$ as follows:

$$
\begin{aligned}
\phi_{X}(u) & =\phi_{N T S}(u ; \alpha, \theta, \beta, \gamma, \mu) \\
& =\exp \left((\mu-\beta) u i-\frac{2 \theta^{1-\frac{\alpha}{2}}}{\alpha}\left(\left(\theta-i \beta u+\frac{\gamma^{2} u^{2}}{2}\right)^{\frac{\alpha}{2}}-\theta^{\frac{\alpha}{2}}\right)\right) .
\end{aligned}
$$

The mean of $X$ is equal to $E[X]=\mu$, and $\operatorname{Var}(X)=\gamma^{2}+\beta^{2}\left(\frac{2-\alpha}{2 \theta}\right)$, respectively. Moreover we can prove that $E\left[e^{u X}\right]<\infty$ if and only if

$$
u \in\left[\frac{-\beta-\sqrt{\beta^{2}+2 \gamma^{2} \theta}}{\gamma^{2}}, \frac{-\beta+\sqrt{\beta^{2}+2 \gamma^{2} \theta}}{\gamma^{2}}\right] .
$$

Providing $\mu=0$ and $\gamma=\sqrt{1-\beta^{2}\left(\frac{2-\alpha}{2 \theta}\right)}$ with $|\beta|<\sqrt{\frac{2 \theta}{2-\alpha}}$, the NTS random variable has zero mean and unit variance. In this case, $X$ is referred to as the standard NTS random variable with parameters $(\alpha, \theta, \beta)$ which we denote by $X \sim$ $\operatorname{stdNTS}(\alpha, \theta, \beta) .{ }^{5}$ The characteristic function of $X$ is equal to

$\phi_{X}(u)=\exp \left(-\beta u i-\frac{2 \theta^{1-\frac{\alpha}{2}}}{\alpha}\left(\left(\theta-i \beta u+\left(1-\frac{\beta^{2}(2-\alpha)}{2 \theta}\right) \frac{u^{2}}{2}\right)^{\frac{\alpha}{2}}-\theta^{\frac{\alpha}{2}}\right)\right)$.

Let $X=\left(X_{1}, X_{2}, \cdots, X_{N}\right)^{T}$ be a multivariate random variable given by

$$
X=\mu+\beta(T-1)+\gamma \sqrt{T} \varepsilon,
$$

where

$$
\begin{aligned}
& \mu=\left(\mu_{1}, \mu_{2}, \cdots, \mu_{N}\right)^{T} \in \mathbb{R}^{N} \\
& \beta=\left(\beta_{1}, \beta_{2}, \cdots, \beta_{N}\right)^{T} \in \mathbb{R}^{N} \\
& \gamma=\left(\gamma_{1}, \gamma_{2}, \cdots, \gamma_{N}\right)^{T} \in \mathbb{R}_{+}^{N} \text { with } \mathbb{R}_{+}=[0, \infty) \\
& \varepsilon=\left(\varepsilon_{1}, \varepsilon_{2}, \cdots, \varepsilon_{N}\right)^{T} \text { is } N \text {-dim standard normal distribution with a covari- } \\
& \text { ance matrix } \rho \text {. That is, } \varepsilon_{n} \sim N(0,1) \text { for } n \in\{1,2, \cdots, N\} \text { and }(k, l) \text {-th } \\
& \text { element of } \rho \text { is given by } \rho_{k, l}=\operatorname{cov}\left(\varepsilon_{k}, \varepsilon_{l}\right) \text { for } k, l \in\{1,2, \cdots, N\} .
\end{aligned}
$$

\footnotetext{
${ }^{5}$ See Rachev et al (2011) for more details.
} 
$T=$ the CTS subordinator with parameters $(\alpha, \theta), \alpha \in(0,1)$ and $\theta>0$, such that $T$ is independent to $\varepsilon_{n}$ for all $n=2, \cdots, N$.

Then the random variable $X$ is referred to as the multivariate NTS (MNTS) random variable with parameters $(\alpha, \theta, \beta, \gamma, \mu, \rho)$ which we denote by $X \sim \operatorname{MNTS}(\alpha$, $\theta, \beta, \gamma, \mu, \rho)$. The mean and variance of $X$ are equal to $E[X]=\mu$, and covariance between $X_{k}$ and $X_{l}$ is given by

$$
\operatorname{cov}\left(X_{k}, X_{l}\right)=\rho_{k, l} \gamma_{k} \gamma_{l}+\beta_{k} \beta_{l}\left(\frac{2-\alpha}{2 \theta}\right)
$$

for $k, l \in\{1,2, \cdots, N\}$.

Proposition 1. Let $x=\left(x_{1}, x_{2}, \cdots, x_{N}\right)^{T} \in \mathbb{R}^{N}$. Then $x^{T} X \sim \operatorname{NTS}(\alpha, \theta, \bar{\beta}, \bar{\gamma}, \bar{\mu})$, where

$$
\bar{\mu}=\sum_{n=1}^{N} x_{n} \mu_{n}, \quad \bar{\beta}=\sum_{n=1}^{N} x_{n} \beta_{n}, \text { and } \bar{\gamma}=\sqrt{\sum_{k=1}^{N} \sum_{l=1}^{N} x_{k} x_{l} \gamma_{k} \gamma_{l} \rho_{k, l}}
$$

Proof. The random variable $x^{T} X$ can be represented by

$$
x^{T} X=\sum_{n=1}^{N} x_{n} X_{n}=\sum_{n=1}^{N} x_{n} \mu_{n}+\left(\sum_{n=1}^{N} x_{n} \beta_{n}\right)(T-1)+\sqrt{T}\left(\sum_{n=1}^{N} x_{n} \gamma_{n} \varepsilon_{n}\right) .
$$

Since we have

$$
\sum_{n=1}^{N} x_{n} \gamma_{n} \varepsilon_{n}=\xi \sqrt{\sum_{k=1}^{N} \sum_{l=1}^{N} x_{k} x_{l} \gamma_{k} \gamma_{l} \rho_{k, l},}, \quad \sim N(0,1)
$$

by the property of linear combination of normal random variables, we obtain the result by the definition of NTS distribution.

Providing $\mu_{n}=0$ and $\gamma_{n}=\sqrt{1-\beta_{n}^{2}\left(\frac{2-\alpha}{2 \theta}\right)}$ with $\left|\beta_{n}\right|<\sqrt{\frac{2 \theta}{2-\alpha}}$ for all $n$ $\in\{1,2, \cdots, N\}$, the MNTS random variable $X$ has $E[X]=(0,0, \cdots, 0)^{T}$ and $\operatorname{Var}(X)=(1,1, \cdots, 1)^{T}$. In this case, $X$ is referred to as the standard MNTS random variable with parameters $(\alpha, \theta, \beta, \rho)$ and we denote it by $X \sim \operatorname{stdMNTS}(\alpha, \theta, \beta, \rho)$.

\section{Marginal contributions of VaR and AVaR}

In this paper, we assume that (1) $R=\left(R_{1}, R_{2}, \cdots, R_{N}\right)^{T}$ is a random vector of returns for $N$ stocks, (2) $R_{p}(w)=w^{T} \cdot R$ is a portfolio return for a weight 
vector $w=\left(w_{1}, w_{2}, \cdots, w_{N}\right)^{T}$, and (3) $Z=R_{j}, Y=\sum_{n=1, n \neq j}^{N} w_{n} R_{n}$ for $j \in\{1,2, \cdots, N\}$. Moreover, let $\phi_{R_{p}(w)}$ denote the characteristic function of $R_{p}(w)$, and $f_{(Y, Z)}(u, v)$ and $\phi_{(Y, Z)}(u, v)$ be the density and characteristic functions of $(Y, Z)$, respectively. Then clearly we have $R_{p}(w)=Y+w_{j} Z$.

The definition of $\operatorname{VaR}$ for $R_{p}(w)$ with the significance level $\eta$ is

$$
\operatorname{VaR}_{\eta}\left(R_{p}(w)\right)=-\inf \left\{x \mid \mathbb{P}\left[R_{p}(w) \leq x\right]>\eta\right\} .
$$

If $R_{p}(w)$ is continuous, then we have

$$
\operatorname{VaR}_{\eta}\left(R_{p}(w)\right)=-F_{R_{p}(w)}^{-1}(\eta)
$$

where $F_{R_{p}(w)}^{-1}$ is the inverse function of the cumulative distribution function of $R_{p}(w)$. The first derivative of $\operatorname{VaR}_{\eta}\left(R_{p}(w)\right)$ with respect to $w_{j}$, the $j$-th element of $w$, is referred to as the marginal VaR. The marginal VaR is computed by the following proposition whose proof is provided in the appendix to this paper.

Proposition 2. Assume that $\mathbb{P}\left[R_{P}(w)=-\operatorname{VaR}_{\eta}\left(R_{P}(w)\right)\right] \neq 0$ and the random variables $Y$ and $Z$ are continuous and infinitely divisible. If there are $b<0$ and $c>0$ in $\mathbb{R}$ such that $\left|\phi_{(Y, Z)}(u, v+i b)\right|<\infty$ and $\left|\phi_{(Y, Z)}(u, v+i c)\right|<\infty$ for all $(u, v) \in \mathbb{R}^{2}$, then we have

$$
\frac{\partial}{\partial w_{j}} \operatorname{VaR}_{\eta}\left(R_{p}(w)\right)=\frac{I_{1}\left(-\operatorname{VaR}_{\eta}\left(R_{p}(w)\right), b, c\right)}{I_{2}\left(-\operatorname{VaR}_{\eta}\left(R_{p}(w)\right), b, c\right)},
$$

where

$$
\begin{aligned}
& I_{1}(K, b, c)=\int_{-\infty}^{\infty} \int_{-\infty}^{\infty} e^{-i K u}\left(\frac{\phi_{(Y, Z)}(u, v+i c)}{\left(w_{j} u-v-i c\right)^{2}}-\frac{\phi_{(Y, Z)}(u, v+i b)}{\left(v-w_{j} u+i b\right)^{2}}\right) d v d u \\
& I_{2}(K, b, c)=\int_{-\infty}^{\infty} \int_{-\infty}^{\infty} i e^{-i K u}\left(\frac{\phi_{(Y, Z)}(u, v+i c)}{w_{j} u-v-i c}+\frac{\phi_{(Y, Z)}(u, v+i b)}{v-w_{j} u+i b}\right) d v d u
\end{aligned}
$$

The definition of AVaR for $R_{p}(w)$ with the significance level $\eta$ is

$$
\operatorname{AVaR}_{\eta}\left(R_{p}(w)\right)=\frac{1}{\eta} \int_{0}^{\eta} \operatorname{VaR}_{x}\left(R_{p}(w)\right) d x .
$$

If $R_{p}(w)$ is continuous, then we have

$$
\operatorname{AVaR}_{\eta}\left(R_{p}(w)\right)=-E\left[R_{p}(w) \mid R_{p}(w)<-\operatorname{VaR}_{\eta}\left(R_{p}(w)\right)\right] .
$$

Suppose $R_{p}(w)$ is infinitely divisible and the distribution function of $R_{p}(w)$ is continuous. If there is $\delta>0$ such that $\left|\phi_{R_{p}(w)}(-u+i \delta)\right|<\infty$ for all $u \in \mathbb{R}$, then 
the following formula for $\operatorname{AVaR}_{\eta}\left(R_{p}(w)\right)$ can be obtained: ${ }^{6}$

$$
\begin{aligned}
& \operatorname{AVaR}_{\eta}\left(R_{p}(w)\right) \\
& =\operatorname{VaR}_{\eta}\left(R_{p}(w)\right)-\frac{e^{-\operatorname{VaR}_{\eta}\left(R_{p}(w)\right) \delta}}{\pi \eta} \Re \int_{0}^{\infty} e^{-i u \operatorname{VaR}_{\eta}\left(R_{p}(w)\right)} \frac{\phi_{R_{p}(w)}(-u+i \delta)}{(-u+i \delta)^{2}} d u,
\end{aligned}
$$

The first derivative of $\operatorname{AVaR}_{\eta}\left(R_{p}(w)\right)$ with respect to $w_{j}$ is referred to as the marginal AVaR. By taking the first derivative of equation (6) with respect to $w_{j}$, we easily obtain the marginal AVaR as the following proposition without proof:

Proposition 3. Suppose $R_{p}(w)$ is infinitely divisible and the distribution function of $R_{p}(w)$ is continuous. If there is $\delta>0$ such that $\left|\phi_{R_{p}(w)}(-u+i \delta)\right|<\infty$ for all $u \in \mathbb{R}$, then the marginal AVaR with respect to $R_{p}(w)$ is equal to

$$
\begin{aligned}
& \frac{\partial}{\partial w_{j}} \operatorname{AVaR}_{\eta}\left(R_{p}(w)\right)=\frac{\partial}{\partial w_{j}} \operatorname{VaR}_{\eta}\left(R_{p}(w)\right) \\
& \quad+\frac{e^{-\delta \operatorname{VaR}_{\eta}\left(R_{p}(w)\right)}}{\pi \eta} \Re \int_{0}^{\infty} e^{-i u \operatorname{VaR}_{\eta}\left(R_{p}(w)\right)} \frac{\phi_{R_{p}(w)}(-u+i \delta)}{(-u+i \delta)^{2}} \\
& \quad \times\left((\delta+i u) \frac{\partial}{\partial w_{j}} \operatorname{VaR}_{\eta}\left(R_{p}(w)\right)-\frac{\partial}{\partial w_{j}} \log \phi_{R_{p}(w)}(-u+i \delta)\right) d u .
\end{aligned}
$$

Suppose a portfolio weight vector $w=\left(w_{1}, w_{2}, \cdots, w_{N}\right)^{T}$ is given and let $\Delta w=\left(\Delta w_{1}, \Delta w_{2}, \cdots, \Delta w_{N}\right)^{T} \in D$ where $D$ is a zero neighborhood in $\mathbb{R}^{N}$. The optimal portfolios with respect to VaR and AVaR are obtained by solving the following problem:

$$
\begin{aligned}
& \max E\left[R_{p}(\Delta w)\right] \\
& \text { subject to } \Delta \operatorname{VaR}_{\eta}\left(R_{p}(w)\right) \leq 0 \text { and } \sum_{j=1}^{N} \Delta w_{j}=0 .
\end{aligned}
$$

and

$$
\begin{aligned}
& \max E\left[R_{p}(\Delta w)\right] \\
& \text { subject to } \Delta \operatorname{AVaR}_{\eta}\left(R_{p}(w)\right) \leq 0 \text { and } \sum_{j=1}^{N} \Delta w_{j}=0
\end{aligned}
$$

\footnotetext{
${ }^{6}$ See Proposition 2 in Kim et al (2010). In their paper, AVaR is defined on the loss distribution. We modified the proposition here because we are looking at the return distribution instead of the loss distribution.
} 
where

$$
\begin{aligned}
& \Delta \operatorname{VaR}_{\eta}\left(R_{p}(w)\right)=\operatorname{VaR}_{\eta}\left(R_{p}(w+\Delta w)\right)-\operatorname{VaR}_{\eta}\left(R_{p}(w)\right) \\
& \Delta \operatorname{AVaR}_{\eta}\left(R_{p}(w)\right)=\operatorname{AVaR}_{\eta}\left(R_{p}(w+\Delta w)\right)-\operatorname{AVaR}_{\eta}\left(R_{p}(w)\right) .
\end{aligned}
$$

Since we have

$$
\begin{aligned}
& \Delta \operatorname{VaR}_{\eta}\left(R_{p}(w)\right) \approx \sum_{j=1}^{N} \frac{\partial}{\partial w_{j}} \operatorname{VaR}_{\eta}\left(R_{p}(w)\right) \Delta w_{j}, \\
& \Delta \operatorname{AVaR}_{\eta}\left(R_{p}(w)\right) \approx \sum_{j=1}^{N} \frac{\partial}{\partial w_{j}} \operatorname{AVaR}_{\eta}\left(R_{p}(w)\right) \Delta w_{j} .
\end{aligned}
$$

and $E\left[R_{p}(w+\Delta w)\right]-E\left[R_{p}(w)\right]=E\left[R_{p}(\Delta w)\right]=\mu^{T} \cdot \Delta w$, we can find the optimal portfolio on the local domain $D$ with respect to VaR and AVaR, respectively, as follows:

$$
\begin{aligned}
& \Delta w^{*}=\arg \max _{\Delta w \in D} \mu^{T} \cdot \Delta w \\
& \text { subject to } \Delta \operatorname{VaR}_{\eta}\left(R_{p}(w)\right) \leq 0 \text { and } \sum_{j=1}^{N} \Delta w_{j}=0,
\end{aligned}
$$

and

$$
\begin{aligned}
& \Delta w^{*}=\arg \max _{\Delta w \in D} \mu^{T} \cdot \Delta w \\
& \text { subject to } \Delta \operatorname{AVaR}_{\eta}\left(R_{p}(w)\right) \leq 0 \text { and } \sum_{j=1}^{N} \Delta w_{j}=0 .
\end{aligned}
$$

\section{Marginal VaR and marginal AVaR for MNTS model}

We say that the random vector $R$ for $N$ stocks follows the MNTS model if we have

$$
R=\mu+\sigma X,
$$

where $X \sim \operatorname{stdMNTS}(\alpha, \theta, \beta, \rho), \mu=\left(\mu_{1}, \mu_{2}, \cdots, \mu_{N}\right)^{T} \in \mathbb{R}^{N}$, and $\sigma=\left(\sigma_{1}\right.$, $\left.\sigma_{2}, \cdots, \sigma_{N}\right)^{T} \in \mathbb{R}_{+}^{N}$. Under the MNTS model, we have $R_{p}(w) \sim \operatorname{MNTS}(\alpha, \theta$, $\sigma * \beta, \sigma * \gamma, \mu, \rho)$, where $\gamma=\left(\gamma_{1}, \gamma_{2}, \cdots, \gamma_{N}\right)^{T}$ with $\gamma_{n}=\sqrt{1-\beta_{n}^{2}\left(\frac{2-\alpha}{2 \theta}\right)}$ for $n \in\{1,2, \cdots, N\}$, and $*$ is the element-wise product. ${ }^{7}$ Moreover, we have

${ }^{7}$ The element-wise product is defined as

$$
\left(a_{1}, a_{2}, \cdots, a_{N}\right)^{T} *\left(b_{1}, b_{2}, \cdots, b_{N}\right)^{T}=\left(a_{1} b_{1}, a_{2} b_{2}, \cdots, a_{N} b_{N}\right)^{T} .
$$


$E[R]=\mu, \operatorname{Var}(R)=\sigma$ and $\operatorname{cov}\left(R_{k}, R_{l}\right)=\sigma_{k} \sigma_{l} \operatorname{cov}\left(X_{k}, X_{l}\right)$ with $\operatorname{cov}\left(X_{k}, X_{l}\right)=$ $\rho_{k, l} \gamma_{k} \gamma_{l}+\beta_{k} \beta_{l}\left(\frac{2-\alpha}{2 \theta}\right)$ for $k, l \in\{1,2, \cdots, N\}$.

The portfolio return $R_{p}(w)$ for the weight vector $w=\left(w_{1}, w_{2}, \cdots, w_{N}\right)^{T}$ is given by the linear combination of $w$ and $R$ as follows

$R_{p}(w)=w^{T} R=\sum_{n=1}^{N} w_{n} \mu_{n}+\left(\sum_{n=1}^{N} w_{n} \sigma_{n} \beta_{n}\right)(T-1)+\sqrt{T}\left(\sum_{n=1}^{N} w_{n} \sigma_{n} \gamma_{n} \varepsilon_{n}\right)$.

By Proposition 1, we obtain

$$
R_{p}(w) \sim \operatorname{NTS}\left(\alpha, \theta, \beta_{p}, \gamma_{p}, \mu_{p}\right)
$$

where

$$
\mu_{p}=\sum_{n=1}^{N} w_{n} \mu_{n}, \quad \beta_{p}=\sum_{n=1}^{N} w_{n} \sigma_{n} \beta_{n}, \gamma_{p}=\sqrt{\sum_{k=1}^{N} \sum_{l=1}^{N} w_{k} w_{l} \sigma_{k} \sigma_{l} \gamma_{k} \gamma_{l} \rho_{k, l}}
$$

Hence, the characteristic function of $R_{p}(w)$ is given by

$$
\begin{aligned}
& \phi_{R_{p}(w)}(u)=\exp \left[\sum_{n=1}^{N} w_{n}\left(\mu_{n}-\sigma_{n} \beta_{n}\right) u i\right. \\
& \left.-\frac{2 \theta^{1-\frac{\alpha}{2}}}{\alpha}\left(\left(\theta-i u \sum_{n=1}^{N} w_{n} \sigma_{n} \beta_{n}+\frac{u^{2}}{2} \sum_{k=1}^{n} \sum_{l=1}^{n} w_{k} w_{l} \sigma_{k} \sigma_{l} \gamma_{k} \gamma_{l} \rho_{k, l}\right)^{\frac{\alpha}{2}}-\theta^{\frac{\alpha}{2}}\right)\right]
\end{aligned}
$$

The first-order partial derivative of the characteristic function of $R_{p}(w)$ for $w_{j}$ is equal to

$$
\frac{\partial}{\partial w_{j}} \phi_{R_{p}(w)}(u)=\phi_{R_{p}(w)}(u) \frac{\partial}{\partial w_{j}} \log \phi_{R_{p}(w)}(u)
$$

where

$$
\begin{aligned}
& \frac{\partial}{\partial w_{j}} \log \phi_{R_{p}(w)}(u) \\
&=\left(\mu_{j}-\sigma_{j} \beta_{j}\right) u i+\left(1-\frac{i u}{\theta} \sum_{n=1}^{N} w_{n} \sigma_{n} \beta_{n}+\frac{u^{2}}{2 \theta} \sum_{k=1}^{n} \sum_{l=1}^{n} w_{k} w_{l} \sigma_{k} \sigma_{l} \gamma_{k} \gamma_{l} \rho_{k, l}\right)^{\frac{\alpha}{2}-1} \\
& \times\left(u i \sigma_{j} \beta_{j}-u^{2} \sigma_{j} \gamma_{j} \sum_{n=1}^{N} w_{n} \sigma_{n} \gamma_{n} \rho_{n, j}\right)
\end{aligned}
$$


for $j \in\{1,2, \cdots, N\}$

Under the MNTS model, the values $Z=R_{j}$ and $Y=\sum_{n=1, n \neq j}^{N} w_{n} R_{n}$ for $j \in\{1,2, \cdots, N\}$ are equal to

$$
\begin{aligned}
& Z=R_{j}=\mu_{Z}+\beta_{Z}(T-1)+\gamma_{Z} \sqrt{T} \varepsilon_{Z} \\
& \text { and } Y=\mu_{Y}+\beta_{Y}(T-1)+\gamma_{Y} \sqrt{T} \varepsilon_{Y},
\end{aligned}
$$

where

$$
\begin{aligned}
\mu_{Z} & =\mu_{j}, \beta_{Z}=\sigma_{j} \beta_{j}, \gamma_{Z}=\sigma_{j} \gamma_{j}, \varepsilon_{Z}=\varepsilon_{j} \sim N(0,1), \\
\mu_{Y} & =\sum_{n=1, n \neq j}^{N} w_{n} \mu_{n}, \beta_{Y}=\sum_{n=1, n \neq j}^{N} w_{n} \sigma_{n} \beta_{n}, \\
\gamma_{Y} & =\sqrt{\sum_{k=1, n \neq j}^{N} \sum_{l=1, n \neq j}^{N} w_{k} w_{l} \sigma_{k} \sigma_{l} \gamma_{k} \gamma_{l} \rho_{k, l},}
\end{aligned}
$$

and

$$
\varepsilon_{Y}=\frac{1}{\gamma_{Y}} \sum_{k=1, n \neq j}^{N} w_{k} \sigma_{k} \gamma_{k} \varepsilon_{k} \sim N(0,1) .
$$

Moreover, we have

$$
\rho_{Y, Z}=\operatorname{cov}\left(\varepsilon_{Y}, \varepsilon_{Z}\right)=\frac{1}{\gamma_{Y}} \sum_{k=1, n \neq j}^{N} w_{k} \sigma_{k} \gamma_{k} \rho_{k, j},
$$

and hence we have

$$
(Y, Z) \sim \operatorname{MNTS}\left(\alpha, \theta,\left(\begin{array}{c}
\beta_{Y} \\
\beta_{Z}
\end{array}\right),\left(\begin{array}{l}
\gamma_{Y} \\
\gamma_{Z}
\end{array}\right),\left(\begin{array}{l}
\mu_{Y} \\
\mu_{Z}
\end{array}\right),\left(\begin{array}{cc}
1 & \rho_{Y, Z} \\
\rho_{Y, Z} & 1
\end{array}\right)\right)
$$

Since $u Z+v Y$ is a linear combination of $(Y, Z)$ for $(u, v) \in \mathbb{R}, u Z+v Y$ becomes a NTS random variable; that is,

$$
\begin{aligned}
& u Z+v Y \\
& \sim \operatorname{NTS}\left(\alpha, \theta, u \beta_{Y}+v \beta_{Z}, \sqrt{u^{2} \gamma_{Y}^{2}+2 u v \rho_{Z, Y} \gamma_{Y} \gamma_{Z}+v^{2} \gamma_{Z}^{2}}, u \mu_{Y}+v \mu_{Z}\right)
\end{aligned}
$$


by Proposition 1 . Hence the characteristic function of $(Y, Z)$ is given by

$$
\begin{aligned}
& \phi_{(Y, Z)}(u, v)=E\left[e^{i(u Y+v Z)}\right] \\
& =\exp \left[i u\left(\mu_{Y}-\beta_{Y}\right)+i v\left(\mu_{Z}-\beta_{Z}\right)\right. \\
& \left.-\frac{2 \theta^{1-\frac{\alpha}{2}}}{\alpha}\left(\left(\theta-i\left(u \beta_{Y}+v \beta_{Z}\right)+\frac{1}{2} \sqrt{u^{2} \gamma_{Y}^{2}+2 u v \rho_{Y, Z} \gamma_{Y} \gamma_{Z}+v^{2} \gamma_{Z}^{2}}\right)^{\frac{\alpha}{2}}-\theta^{\frac{\alpha}{2}}\right)\right] .
\end{aligned}
$$

We can prove that $\phi_{(Y, Z)}(u, v+i a)<\infty$ if $a \in\left[\frac{\beta_{Z}-\sqrt{\beta_{Z}^{2}+2 \gamma_{Z}^{2} \theta}}{\gamma_{Z}^{2}}, \frac{\beta_{Z}+\sqrt{\beta_{Z}^{2}+2 \gamma_{Z}^{2} \theta}}{\gamma_{Z}^{2}}\right]$. Therefore, by substituting (16) into (5) with $b$ and $c$ such that

$$
\frac{\beta_{Z}-\sqrt{\beta_{Z}^{2}+2 \gamma_{Z}^{2} \theta}}{\gamma_{Z}^{2}} \leq b<0<c \leq \frac{\beta_{Z}+\sqrt{\beta_{Z}^{2}+2 \gamma_{Z}^{2} \theta}}{\gamma_{Z}^{2}},
$$

we obtain the marginal $\mathrm{VaR}$ with respect to the $j$-th stock in the portfolio under the MNTS model. Moreover, by substituting (13) and (15) into (7), we obtain the marginal AVaR with respect to the $j$-th stock in the portfolio.

\section{Empirical illustration}

In this section, we report the maximum likelihood estimation (MLE) of the MNTS model using data obtained from OptionMetricss Ivy DB in the Wharton Research Data Services. In our empirical study, we use historical prices for 29 of the 30 component stocks comprising the Dow Jones Industrial Average (DJIA) as of October $2010 .^{8}$ The name of the 29 stocks and their tickers are listed in Table 1. First, we take the stock prices for the 29 companies from November 1, 1999 to October 31, 2009. The number of daily returns for each stock and the index is 2,516 . Then we form an equally weighted portfolio with those 29 stocks, calculating the marginal VaR and marginal AVaR with respect to each stock in the portfolio. Finally, we find the optimal portfolio which locally minimizes VaR and AVaR.

We assume that daily returns of the 29 stocks follow the MNTS model. That is $R=\mu+\sigma X$ where $\mu$ and $\sigma$ are the mean vector and the standard deviation vector for those 29 stock returns, and $X$ is the 29-dimensional standard MNTS random vector with parameters $(\alpha, \theta, \beta, \rho)$. We estimate $\mu$ and $\sigma$ by the sample mean $\hat{\mu}$

\footnotetext{
${ }^{8}$ Kraft Foods (KFT) is excluded because the time series we employ begins in 1999 but this company was not listed until 2001.
} 
and the sample standard deviation $\hat{\sigma}$ for the historical data. Parameters $\alpha$ and $\theta$, which are obtained from the CTS subordinator, are estimated using the DJIA data. That is, we assume that normalized daily returns for the DJIA follows the standard NTS distribution, and fit those parameters using the MLE. The estimated values using this method are presented in Table 2. We use the Kolmogorov-Smirnov (KS) and Anderson-Darling (AD) goodness-of-fit tests. Then, we fit the standard MNTS parameter by MLE using normalizing data for the 29 stock returns.

For each $j$-th stock $(j=1,2, \cdots, 29)$ we assume that the normalized return follows the standard NTS distribution with parameter $\left(\alpha, \theta, \beta_{j}\right)$ and estimate $\beta_{j}$ using the MLE with $\alpha=\hat{\alpha}$ and $\theta=\hat{\theta}$ where $\hat{\alpha}$ and $\hat{\theta}$ are reported in Table 2. $\hat{\beta}_{j}$ denotes the estimated value of $\beta_{j}$ and let $\hat{\gamma}_{j}=\sqrt{1-\hat{\beta}_{j}^{2}\left(\frac{2-\hat{\alpha}}{2 \hat{\theta}}\right)}$. The estimated parameters are presented in Table 3 along with the goodness-of-fit test statistic. Based on the KS test, only the estimated parameters for six of the 29 stocks are rejected at the $1 \%$ significance level. None of the parameters for the stocks are rejected based on the AD test at the $1 \%$ significance level. The matrix $\rho$ is estimated by (4). That is,

$$
\hat{\rho}_{k, l}=\frac{\widehat{\operatorname{cov}}\left(X_{k}, X_{l}\right)-\hat{\beta}_{k} \hat{\beta}_{l}\left(\frac{2-\hat{\alpha}}{2 \hat{\theta}}\right)}{\hat{\gamma}_{k} \hat{\gamma}_{l}}
$$

for $k, l \in\{1,2, \cdots, 29\}$, where $\widehat{\operatorname{cov}}\left(X_{k}, X_{l}\right)$ is the sample covariation between the $k$-th stock and the $l$-th stock returns. Matrix $\hat{\rho}$, which is the estimated matrix for $\rho$, is presented in Table 4 .

Consider an equally weight portfolio for the 29 stocks. Then the portfolio return $R_{p}(w)$ is equal to $\sum_{j=1}^{29} w_{j} R_{j}$ where $w_{j}=1 / 29$ and $R_{j}$ is the random variable for the daily return of the $j$-th stock in the portfolio. Under the MNTS model, $R_{p}(w)$ becomes the 1-dim NTS distributed random variable by Proposition 1. If we assume that $R=\mu+\sigma X$ where $X \sim N(0, \Sigma)$, then we obtain the normal model. In the normal model, the portfolio return $R_{p}(w)$ follows a normal distribution with mean $\mu$ and variance $(w * \sigma)^{T} \Sigma(w * \sigma)$.

Using the estimated parameters in Table 3 and the matrix $\hat{\rho}$ in Table 4, we obtain

$$
R_{p}(w) \sim \operatorname{NTS}(\hat{\alpha}, \hat{\theta}, \hat{\bar{\beta}}, \hat{\bar{\gamma}}, \hat{\bar{\mu}})
$$

where $\hat{\alpha}=1.0301, \hat{\theta}=0.2205, \hat{\bar{\beta}}=4.7763 \cdot 10^{-4}, \hat{\bar{\gamma}}=0.0142$, and $\hat{\bar{\mu}}=$ $2.7788 \cdot 10^{-4}$ by parameterization of Proposition 1 . The probability density functions for the NTS, normal, and empirical distributions are presented in Figure $1 .^{9}$ Comparing the empirical density to the NTS and normal density in Figure 1, we find that the portfolio distribution assuming the normal model is not similar to the

\footnotetext{
${ }^{9}$ The NTS density function is computed by the fast Fourier transform method explained in Rachev et al (2011).
} 
empirical distribution, while the portfolio distribution assuming the MNTS model is very similar to the empirical distribution. More precisely, we show the Q-Q plots in Figure 2. The empirical density deviates significantly from the normal density as can be seen from the first Q-Q plots in Figure 2. This deviation almost completely disappears when we use the MNTS model.

Applying the KS and AD tests to the difference between the empirical portfolio return distribution and the portfolio distribution of the MNTS model, we obtain $K S=0.0264$ with $p$-value 0.0588 and $A D=0.0562$ with $p$-value 0.9999 . For both the KS and AD tests, the portfolio distribution of the MNTS model is not rejected at the $1 \%$ significance level. The KS statistic applied to the empirical portfolio return distribution and the portfolio distribution of the normal model is 0.4753 with a $p$-value of zero. ${ }^{10}$ Hence, the portfolio distribution of the normal model is rejected at $1 \%$ significant level by the KS test.

The VaR and the AVaR values for the equally weight portfolio for the 29 stocks at confidence levels $\{0.1 \%, 0.2 \%, \cdots, 1 \%, \cdots, 5 \%\}$ are exhibited in the left (for $\mathrm{VaR}$ ) and the right (for AVaR) panels of Figure 3. We plot the values of the normal and the MNTS models in the two figures and compare the values to their empirical counterparts. From the results shown in the left panel of Figure 3, both the normal VaR and the MNTS VaR are similar to the empirical VaR if the confidence level exceeds $3 \%$. If the confidence level is less than $2 \%$, the MNTS VaR is still similar to the empirical VaR while the normal VaR is smaller than the empirical VaR. Moreover, from the results shown in the right panel of Figure 3, the AVaR of the MNTS model is relatively similar to the empirical AVaR compared to the normal distribution.

Table 5 provides the marginal VaR and marginal AVaR calculated based on Propositions 2 and 3 for the 29 stocks in the equally weighted portfolio under the MNTS model. As we explained at the end of Section 4, the marginal VaR is obtained by substituting (16) into (5), and the marginal AVaR is obtained by substituting (13) and (15) into (7). As shown in Table 5, five stocks contribute greatly to the portfolio $1 \%$-VaR, and five stocks contribute greatly to the portfolio 1\%-AVaR. Two stocks (AXP and JPM) are common to both.

We refer to the optimal portfolios obtained by (10) and (11) as the VaR-optimal portfolio and the AVaR-optimal portfolio, respectively. Results for these optimal portfolios are also in Table 5 under the local domain

$$
D=\left\{\left(x_{1}, x_{2}, \cdots, x_{29}\right) \mid x_{j} \in(-0.0172,0.0172), j=1,2, \cdots, 29\right\} .
$$

The initial portfolio is the equally weighted portfolio. The $\Delta w^{*}$ by (10) and (11) are located in the fourth and the eighth columns in the table, and $w^{n e w}=w+\Delta w^{*}$ are presented in the fifth and the ninth columns. The expected daily return is

\footnotetext{
${ }^{10} \mathrm{We}$ do not mention the $\mathrm{AD}$ test since we obtained an infinite $\mathrm{AD}$ value for the normal model.
} 
$2.7788 \cdot 10^{-4}$ for the equally weighted portfolio, and the expected daily returns for the VaR-optimal and the AVaR-optimal portfolios are $3.4449 \cdot 10^{-4}$ and 3.5397 . $10^{-4}$, respectively. The relative change for the expected daily return is defined by $\left(\mu^{T} \cdot w^{n e w}-\mu^{T} \cdot w\right) /\left(\mu^{T} \cdot w\right)$. Values of the relative change for the expected daily returns are 0.2397 and 0.2738 with respect to the VaR-optimal and the AVaRoptimal portfolios, respectively. That means the investor obtains a $23.97 \%$ and $27.38 \%$ better expected daily return for the VaR-optimal and the AVaR-optimal portfolios, respectively.

\section{Conclusion}

In this paper, we describe the MNTS distributed market model and derive the closed-form solution for two important measures used by portfolio managers: the marginal VaR and the marginal AVaR. The proposed model is applied to the analysis of an equally weighted portfolio comprised of 29-component stocks of the DJIA. We find that the time series models based on the multivariate normal distributed model is rejected empirically and therefore does not provide a reliable distribution of portfolio returns. Our empirical evidence indicates that the MNTS model provides more realistic results in measuring market risk compared to standard models based on the normal distribution assumption. Finally, after deriving a closed-form solutions of the marginal VaR and the marginal AVaR based on the MNTS model, we applied these formulas to optimize the portfolio with 29 stocks.

\section{Appendix: Proof of Proposition 2}

In order to prove Proposition 2, we need the following lemma.

Lemma. If $\mathbb{P}\left[R_{P}(w)=-\operatorname{Va} R_{\eta}\left(R_{P}(w)\right)\right] \neq 0$, then we have

$$
\frac{\partial}{\partial w_{j}} \operatorname{VaR}_{\eta}\left(R_{p}(w)\right)=\frac{-\int_{-\infty}^{\infty} z f\left(-\operatorname{VaR}_{\eta}\left(R_{p}(w)\right)-w_{j} z, z\right) d z}{\int_{-\infty}^{\infty} f\left(-\operatorname{VaR}_{\eta}\left(R_{p}(w)\right)-w_{j} z, z\right) d z} .
$$

Proof. By the definition of $\operatorname{VaR}_{\eta}\left(R_{p}(w)\right)$, we have

$$
\mathbb{P}\left[Y+w_{j} Z \leq-\operatorname{VaR}_{\eta}\left(R_{p}(w)\right)\right]=\eta
$$

Let $w_{j} \neq 0$,

$$
\int_{-\infty}^{\infty} \int_{-\infty}^{-\operatorname{VaR}_{\eta}\left(R_{p}(w)\right)-w_{j} z} f(y, z) d y d z=\eta
$$


where $f(z, y)$ is the probability density function for $(Y, Z)$. Taking the first derivative with respect to $w_{j}$, we obtain

$$
\int_{-\infty}^{\infty}\left(-\frac{\partial}{\partial w_{j}} \operatorname{VaR}_{\eta}\left(R_{p}(w)\right)-z\right) f\left(-\operatorname{VaR}_{\eta}\left(R_{p}(w)\right)-w_{j} z, z\right) d z=0 .
$$

Hence, we have

$$
\begin{aligned}
& \left(\frac{\partial}{\partial w_{j}} \operatorname{VaR}_{\eta}\left(R_{p}(w)\right)\right) \int_{-\infty}^{\infty} f\left(-\operatorname{VaR}_{\eta}\left(R_{p}(w)\right)-w_{j} z, z\right) d z \\
& =-\int_{-\infty}^{\infty} z f\left(-\operatorname{VaR}_{\eta}\left(R_{p}(w)\right)-w_{j} z, z\right) d z .
\end{aligned}
$$

Since $\int_{-\infty}^{\infty} f\left(-\operatorname{VaR}_{\eta}\left(R_{p}(w)\right)-w_{j} z, z\right) d z=\mathbb{P}\left[Y+w_{j} Z=-\operatorname{VaR}_{\eta}\left(R_{p}(w)\right)\right] \neq 0$, we obtain (17).

Proof of Proposition 2. The pdf of $(Y, Z)$ is obtained by the complex inversion formula as follows:

$$
f(y, z)=\frac{1}{(2 \pi)^{2}} \int_{-\infty}^{\infty} \int_{-\infty}^{\infty} e^{-i(u y+v(z+i a))} \phi_{(Y, Z)}(u, v+i a) d u d v
$$

if $\left|\phi_{(Y, Z)}(u, v+i a)\right|<\infty$ for all $(u, v) \in \mathbb{R}^{2}$. Since $|\phi(u, v+i b)|<\infty$ and $|\phi(u, v-i c)|<\infty$ for all $(u, v) \in \mathbb{R}^{2}$, we obtain

$$
\begin{aligned}
& \int_{-\infty}^{\infty} z f(K-m z, z) d z=\int_{0}^{\infty} z f(K-m z, z) d z+\int_{-\infty}^{0} z f(K-m z, z) d z \\
& =\frac{1}{(2 \pi)^{2}} \int_{0}^{\infty} z \int_{-\infty}^{\infty} \int_{-\infty}^{\infty} e^{-i K u+i z(m u-v-i b)} \phi_{(Y, Z)}(u, v+i b) d u d v d z \\
& \quad+\frac{1}{(2 \pi)^{2}} \int_{-\infty}^{0} z \int_{-\infty}^{\infty} \int_{-\infty}^{\infty} e^{-i K u+i z(m u-v-i c)} \phi_{(Y, Z)}(u, v-i c) d u d v d z \\
& =\frac{1}{(2 \pi)^{2}} \int_{-\infty}^{\infty} \int_{-\infty}^{\infty} e^{-i K u} \phi_{(Y, Z)}(u, v+i b) \int_{0}^{\infty} z e^{-i z(v-m u+i b)} d z d u d v \\
& \quad-\frac{1}{(2 \pi)^{2}} \int_{-\infty}^{\infty} \int_{-\infty}^{\infty} e^{-i K u} \phi_{(Y, Z)}(u, v-i c) \int_{0}^{\infty} z e^{-i z(m u-v-i c)} d z d u d v
\end{aligned}
$$

Since we have $\int_{0}^{\infty} z e^{-i z(m+i a)} d z=-\frac{1}{(m+i a)^{2}}$ if $a<0$ and $b,-c<0$, we have

$$
\begin{aligned}
& \int_{-\infty}^{\infty} z f(K-m z, z) d z \\
& =\frac{1}{(2 \pi)^{2}} \int_{-\infty}^{\infty} \int_{-\infty}^{\infty} e^{-i K u}\left(\frac{\phi_{(Y, Z)}(u, v-i c)}{(m u-v-i c)^{2}}-\frac{\phi_{(Y, Z)}(u, v+i b)}{(v-m u+i b)^{2}}\right) d u d v
\end{aligned}
$$


On the other hand, we have

$$
\begin{aligned}
& \int_{-\infty}^{\infty} f(K-m z, z) d z=\int_{0}^{\infty} f(K-m z, z) d z+\int_{-\infty}^{0} f(K-m z, z) d z \\
& =\frac{1}{(2 \pi)^{2}} \int_{0}^{\infty} \int_{-\infty}^{\infty} \int_{-\infty}^{\infty} e^{-i K u+i z(m u-v-i b)} \phi_{(Y, Z)}(u, v+i b) d u d v d z \\
& \quad+\frac{1}{(2 \pi)^{2}} \int_{-\infty}^{0} \int_{-\infty}^{\infty} \int_{-\infty}^{\infty} e^{-i K u+i z(m u-v-i c)} \phi_{(Y, Z)}(u, v-i c) d u d v d z \\
& =\frac{1}{(2 \pi)^{2}} \int_{-\infty}^{\infty} \int_{-\infty}^{\infty} e^{-i K u} \phi_{(Y, Z)}(u, v+i b) \int_{0}^{\infty} e^{-i z(v-m u+i b)} d z d u d v \\
& \quad+\frac{1}{(2 \pi)^{2}} \int_{-\infty}^{\infty} \int_{-\infty}^{\infty} e^{-i K u} \phi_{(Y, Z)}(u, v-i c) \int_{0}^{\infty} e^{-i z(m u-v-i c)} d z d u d v
\end{aligned}
$$

Since $b<0<c$ and we have $\int_{0}^{\infty} e^{-i z(m+i a)} d z=-\frac{i}{m+i a}$ if $a<0$, we have

$$
\begin{aligned}
& \int_{-\infty}^{\infty} f(K-m z, z) d z \\
& =-\frac{i}{(2 \pi)^{2}} \int_{-\infty}^{\infty} \int_{-\infty}^{\infty} e^{-i K u}\left(\frac{\phi_{(Y, Z)}(u, v-i c)}{m u-v-i c}+\frac{\phi_{(Y, Z)}(u, v+i b)}{v-m u+i b}\right) d u d v
\end{aligned}
$$

By substituting (18) and (19) with $m=w_{j}$ into (17) in the above lemma, we obtain the result. 
Table 1: Names and tickers of the 29 component companies of the DJIA.

\begin{tabular}{llc}
\hline Company Name & Ticker & Index $(j)$ \\
\hline 3M Co. & MMM & 1 \\
Alcoa Inc. & AA & 2 \\
American Express Co. & AXP & 3 \\
AT\&T Inc. & T & 4 \\
Bank of America Corp. & BAC & 5 \\
Boeing Co. & BA & 6 \\
Caterpillar Inc. & CAT & 7 \\
Chevron Corp. & CVX & 8 \\
Cisco Systems Inc. & CSCO & 9 \\
Coca-Cola Co. & KO & 10 \\
E.I. DuPont de Nemours \& Co. & DD & 11 \\
Exxon Mobil Corp. & XOM & 12 \\
General Electric Co. & GE & 13 \\
Hewlett-Packard Co. & HPQ & 14 \\
Home Depot Inc. & HD & 15 \\
Intel Corp. & INTC & 16 \\
International Business Machines Corp. & IBM & 17 \\
Johnson \& Johnson & JNJ & 18 \\
JPMorgan Chase \& Co. & JPM & 19 \\
McDonald's Corp. & MCD & 20 \\
Merck \& Co. Inc. & MRK & 21 \\
Microsoft Corp. & MSFT & 22 \\
Pfizer Inc. & PFE & 23 \\
Procter \& Gamble Co. & PG & 24 \\
Travelers Cos. Inc. & TRV & 25 \\
United Technologies Corp. & UTX & 26 \\
Verizon Communications Inc. & VZ & 27 \\
Wal-Mart Stores Inc. & WMT & 28 \\
Walt Disney Co. & DIS & 29 \\
\hline
\end{tabular}


Table 2: Estimated parameters for the DJIA index return.

\begin{aligned} \hline $\begin{array}{r}\text { Sample mean of daily returns } \\ \text { Sample standard deviation of daily returns }\end{array} & 6.2227 \cdot 10^{-5} \\$\hline Parameters of the standard NTS distribution & Confidence interval \\ \hline$\hat{\alpha}=1.0301 & (0.7583,1.3018) \\ \hat{\theta}=0.2205 & (0.0873,0.3536) \\ \hat{\beta}=-0.0369 & (-0.0807,0.0069) \\$\hline Goodness of fit statistic & $p$-value \\ \hline$K S=0.0124 & (0.8280) \\ A D=0.1046 & (0.99995) \\$\hline & \end{aligned}


Table 3: Estimated parameters for the daily return of 29 component companies of the DJIA from November 1, 1999, to October 31, 2009.

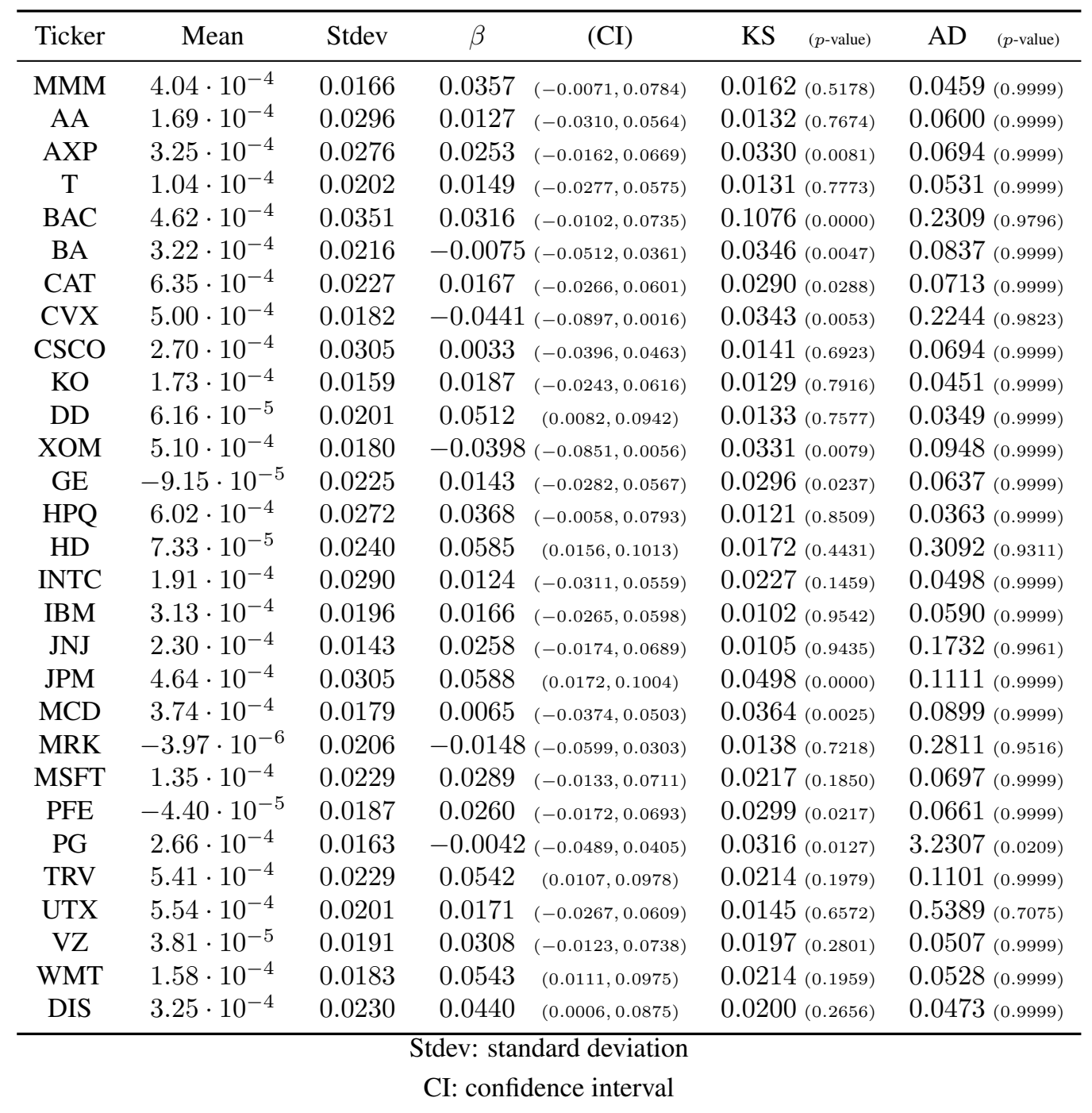




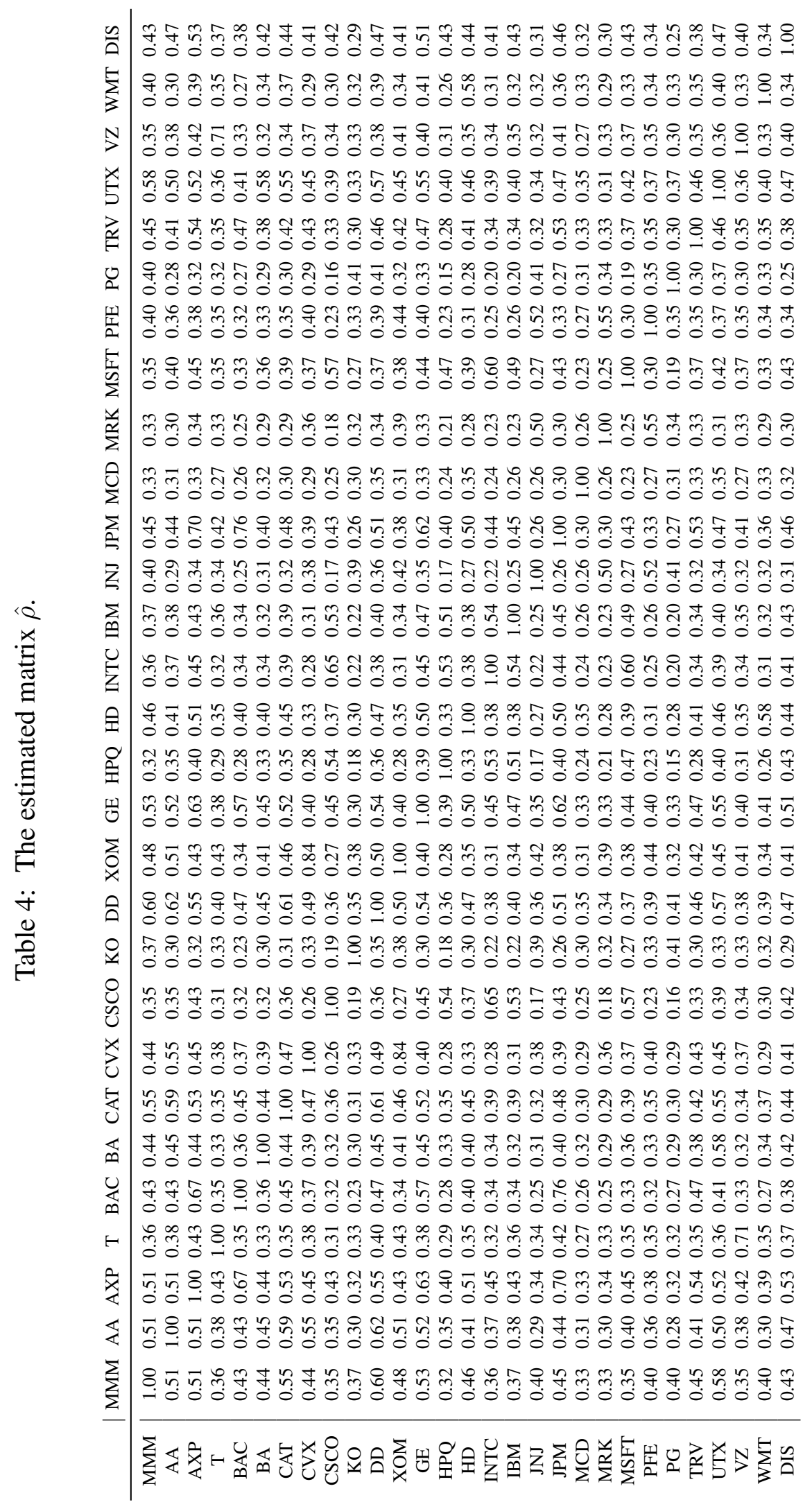


Table 5: Marginal VaR and Marginal AVaR for the equally weighted portfolio consists of 29 stocks, and local optimal portfolios for VaR and AVaR.

\begin{tabular}{|c|c|c|c|c|c|c|c|c|}
\hline \multirow[b]{2}{*}{ TICKER } & \multicolumn{4}{|c|}{ VaR } & \multicolumn{4}{|c|}{ AVaR } \\
\hline & $M V a R_{j}$ & rank & $\Delta w_{j}^{*}$ & $w_{j}^{n e w}$ & $M A V a R_{j}$ & rank & $\Delta w_{j}^{*}$ & $w_{j}^{n e w}$ \\
\hline MMM & 0.0131 & 6 & 0.0043 & 0.0388 & 0.0418 & 22 & 0.0172 & 0.0517 \\
\hline AA & 0.0130 & 7 & -0.0127 & 0.0218 & 0.0785 & 4 & -0.0172 & 0.0172 \\
\hline AXP & 0.0148 & 1 & -0.0070 & 0.0275 & 0.0803 & 3 & -0.0012 & 0.0332 \\
\hline $\mathrm{T}$ & 0.0112 & 18 & -0.0119 & 0.0226 & 0.0458 & 19 & -0.0171 & 0.0174 \\
\hline BAC & 0.0121 & 13 & 0.0116 & 0.0461 & 0.0858 & 1 & 0.0126 & 0.0471 \\
\hline BA & 0.0112 & 19 & 0.0043 & 0.0388 & 0.0523 & 15 & 0.0055 & 0.0400 \\
\hline CAT & 0.0127 & 10 & 0.0172 & 0.0517 & 0.0592 & 8 & 0.0172 & 0.0517 \\
\hline CVX & 0.0111 & 20 & 0.0172 & 0.0517 & 0.0471 & 18 & 0.0172 & 0.0517 \\
\hline CSCO & 0.0110 & 21 & 0.0011 & 0.0356 & 0.0724 & 5 & -0.0054 & 0.0291 \\
\hline KO & 0.0090 & 27 & 0.0004 & 0.0349 & 0.0286 & 28 & -0.0050 & 0.0295 \\
\hline DD & 0.0144 & 4 & -0.0172 & 0.0172 & 0.0530 & 14 & -0.0172 & 0.0172 \\
\hline XOM & 0.0115 & 16 & 0.0172 & 0.0517 & 0.0473 & 17 & 0.0172 & 0.0517 \\
\hline GE & 0.0146 & 2 & -0.0172 & 0.0172 & 0.0643 & 7 & -0.0172 & 0.0172 \\
\hline HPQ & 0.0104 & 24 & 0.0172 & 0.0517 & 0.0566 & 9 & 0.0172 & 0.0517 \\
\hline HD & 0.0129 & 8 & -0.0172 & 0.0172 & 0.0557 & 10 & -0.0172 & 0.0172 \\
\hline INTC & 0.0117 & 14 & -0.0069 & 0.0276 & 0.0701 & 6 & -0.0136 & 0.0209 \\
\hline IBM & 0.0115 & 15 & 0.0026 & 0.0371 & 0.0457 & 20 & 0.0063 & 0.0407 \\
\hline JNJ & 0.0095 & 25 & 0.0029 & 0.0374 & 0.0266 & 29 & 0.0019 & 0.0364 \\
\hline JPM & 0.0144 & 3 & 0.0044 & 0.0389 & 0.0808 & 2 & 0.0141 & 0.0486 \\
\hline MCD & 0.0087 & 28 & 0.0162 & 0.0506 & 0.0331 & 26 & 0.0163 & 0.0508 \\
\hline MRK & 0.0092 & 26 & -0.0133 & 0.0212 & 0.0418 & 23 & -0.0172 & 0.0172 \\
\hline MSFT & 0.0122 & 11 & -0.0127 & 0.0218 & 0.0543 & 12 & -0.0159 & 0.0186 \\
\hline PFE & 0.0108 & 23 & -0.0172 & 0.0172 & 0.0390 & 24 & -0.0172 & 0.0172 \\
\hline PG & 0.0085 & 29 & 0.0085 & 0.0430 & 0.0302 & 27 & 0.0049 & 0.0394 \\
\hline TRV & 0.0122 & 12 & 0.0172 & 0.0517 & 0.0521 & 16 & 0.0172 & 0.0517 \\
\hline UTX & 0.0132 & 5 & 0.0150 & 0.0495 & 0.0537 & 13 & 0.0172 & 0.0517 \\
\hline $\mathrm{VZ}$ & 0.0114 & 17 & -0.0172 & 0.0172 & 0.0420 & 21 & -0.0172 & 0.0172 \\
\hline WMT & 0.0108 & 22 & -0.0065 & 0.0279 & 0.0358 & 25 & -0.0085 & 0.0260 \\
\hline DIS & 0.0127 & 9 & -0.0004 & 0.0341 & 0.0554 & 11 & 0.0052 & 0.0396 \\
\hline
\end{tabular}




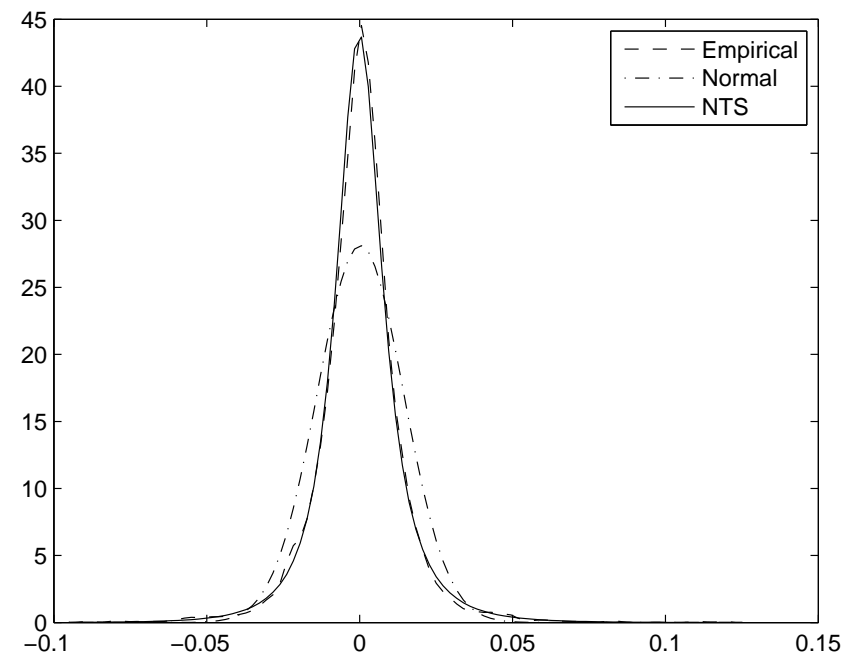

Figure 1: Empirical probability density function for daily portfolio returns and the two probability density functions of the portfolio return obtained by the normal model and the MNTS model.
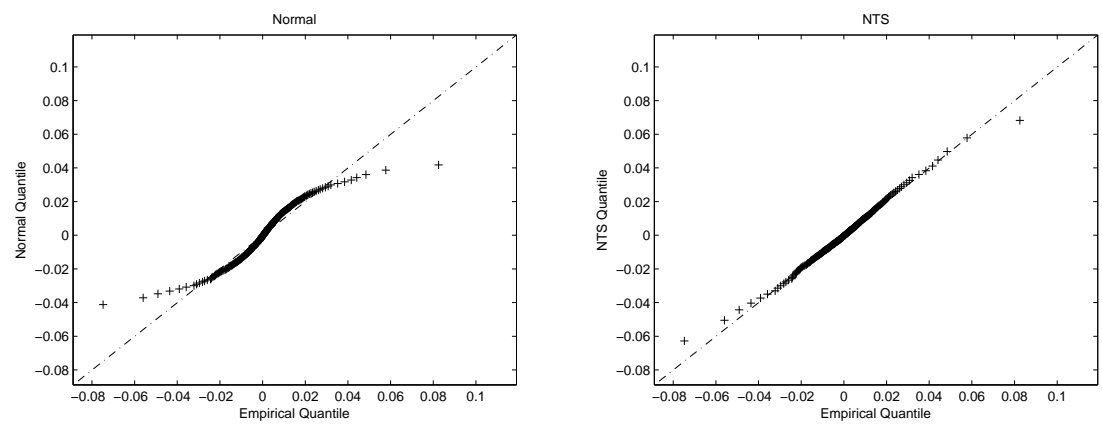

Figure 2: Q-Q plot between the empirical portfolio return distribution and the theoretical portfolio return for the normal model (left) and between the empirical portfolio return distribution and the theoretical portfolio return for the MNTS model (right). 

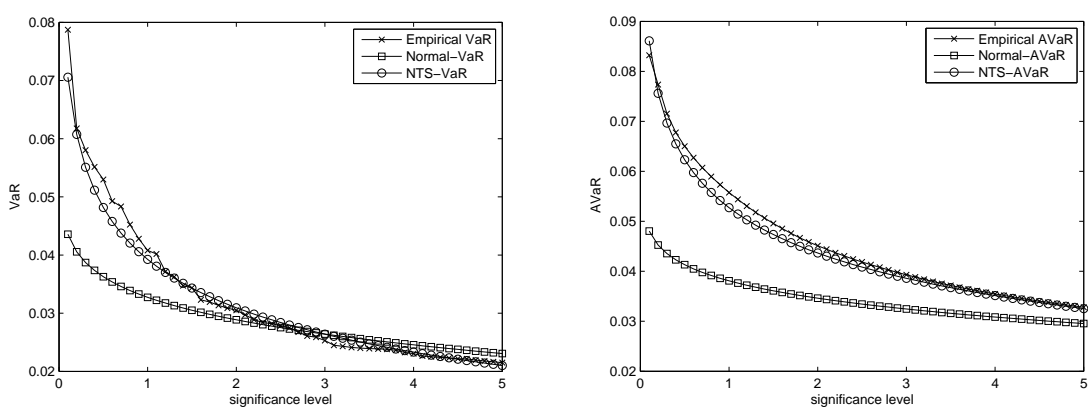

Figure 3: One-day portfolio VaR (left) and portfolio AVaR (right)

\section{References}

Aas K, Hobæk Haff I, Dimakos XK (2006) Risk estimation using the multivariate normal inverse Gaussian distribution. Journal of Risk 8(2):39-60

Adcock C (2010) Asset pricing and portfolio selection based on the multivariate extended skew-Student- $t$ distribution. Annals of Operations Research 176:221234

Barndorff-Nielsen OE, Shephard N (2001) Normal modified stable processes. Economics Series Working Papers from University of Oxford, Department of Economics 72

Dokov S, Stoyanov SV, Rachev ST (2008) Computing VaR and AVaR of skewed-t distribution. Journal of Applied Functional Analysis 3:189-208

Eberlein E, Madan DB (2010) On correlating Lévy processes. Journal of Risk 13(1):3-16

Fabozzi FJ, Gupta F, Markowitz HM (2002) The legacy of modern portfolio theory. Journal of Investing 11(2):7-22

Fama E (1963) Mandelbrot and the stable Paretian hypothesis. Journal of Business $36: 420-429$

Gourieroux C, Laurent J, Scaillet O (2000) Sensitivity analysis of values at risk. Journal of Empirical Finance 7:225-245

Kim YS, Rachev ST, , Bianchi ML, Fabozzi FJ (2010) Computing VaR and AVaR in infinitely divisible distributions. Probability and Mathematical Statistics 30(2):223-245 
Mandelbrot BB (1963a) New methods in statistical economics. Journal of Political Economy 71:421-440

Mandelbrot BB (1963b) The variation of certain speculative prices. Journal of Business 36:394-419

Mansini R, Ogryczak W, Speranza MG (2007) Conditional value at risk and related linear programming models for portfolio optimization. Annals of Operations Research 152:227-256

Markowitz H (1952) Portfolio selection. Journal of Finance 7(1):77-91

Øigård TA, Hanssen A, Hansen RE, Godtliebsen F (2005) EM-estimation and modeling of heavy-tailed processes with the multivariate normal inverse Gaussian distribution. Signal Processing 85:1655-1673

Pflug G (2000) Some remarks on the value-at-risk and the conditional value-atrisk. In: Uryasev S (ed) Probabilistic Constrained Optimization: Methodology and Applications, The Netherlands: Kluwer Academic Publishers, pp 272-281

Rachev ST, Mittnik S (2000) Stable Paretian Models in Finance. John Wiley \& Sons: New York

Rachev ST, Menn C, Fabozzi FJ (2005) Fat-Tailed and Skewed Asset Return Distributions: Implications for Risk Management, Portfolio selection, and Option Pricing. John Wiley \& Sons

Rachev ST, Stoyanov S, Fabozzi FJ (2007) Advanced Stochastic Models, Risk Assessment, and Portfolio Optimization: The Ideal Risk, Uncertainty, and Performance Measures. Hoboken, John Wiley \& Sons: N.J.

Rachev ST, Kim YS, Bianch ML, Fabozzi FJ (2011) Financial Models with Lévy Processes and Volatility Clustering. John Wiley \& Sons

Rockafellar RT, Uryasev S (2000) Optimization of conditional value-at-risk. Journal of Risk 2(3):21-41

Rockafellar RT, Uryasev S (2002) Conditional value-at-risk for general loss distributions. Journal of Banking \& Finance 26:1443-1471

Stoyanov S, Samorodnitsky G, Rachev ST, Ortobelli S (2006) Computing the portfolio conditional value-at-risk in the $\alpha$-stable case. Probability and Mathematical Statistics 26:1-22 
Stoyanov SV, Rachev ST, Fabozzi FJ (2009) Sensitivity of portfolio $\mathrm{VaR}$ and $\mathrm{CVaR}$ to portfolio return characteristics Technical report, Chair of Econometrics, Statistics and Mathematical Finance School of Economics and Business Engineering, KIT, http://statistik.ets.kit.edu/download/technical_reports/distrCharAndRisk_30Nov.pdf 


\section{Working Paper Series in Economics}

recent issues

No. 44 Young Shin Kim, Rosella Giacometti, Svetlozar T. Rachev, Frank J. Fabozzi, Domenico Mignacca: Measuring financial risk and portfolio optimization with a non-Gaussian multivariate model, August 2012

No. 43 Zuodong Lin, Svetlozar T. Rachev, Young Shin Kim, Frank J. Fabozzi: Option pricing with regime switching tempered stable processes, August 2012

No. 42 Siegfried K. Berninghaus, Werner Güth, Stephan Schosser: Backward induction or forward reasoning? An experiment of stochastic alternating offer bargaining, July 2012

No. 41 Siegfried Berninghaus, Werner Güth, King King Li: Approximate truth of perfectness - an experimental test, June 2012

No. 40 Marten Hillebrand and Tomoo Kikuchi: A mechanism for booms and busts in housing prices, May 2012

No. 39 Antje Schimke: Entrepreneurial aging and employment growth in the context of extreme growth events, May 2012

No. 38 Antje Schimke, Nina Teichert, Ingrid Ott: Impact of local knowledge endowment on employment growth in nanotechnology, February 2012

No. 37 Siegfried K. Berninghaus, Lora Todorova, Bodo Vogt: A simple questionnaire can change everything - are strategy choices in coordination games stable?, December 2011

No. 36 Matthias Duschl, Antje Schimke, Thomas Brenner, Dennis Luxen: Firm growth and the spatial impact of geolocated external factors - empirical evidence for German manufacturing firms, November 2011

No. 35 Mirja Meyborg: The impact of West-German universities on regional innovation activities - a social network analysis, October 2011 